\title{
Modular Frobenius pseudo-varieties
}

\section{Aureliano M. Robles-Pérez ${ }^{1}$ (D) - José Carlos Rosales ${ }^{2}$}

Received: 23 February 2021 / Accepted: 27 September 2021 / Published online: 28 October 2021

(c) The Author(s) 2021

\section{Abstract}

If $m \in \mathbb{N} \backslash\{0,1\}$ and $A$ is a finite subset of $\bigcup_{k \in \mathbb{N} \backslash\{0,1\}}\{1, \ldots, m-1\}^{k}$, then we denote by

$$
\begin{gathered}
\mathscr{C}(m, A)=\left\{S \in \mathscr{S}_{m} \mid s_{1}+\cdots+s_{k}-m \in S \text { if }\left(s_{1}, \ldots, s_{k}\right) \in S^{k}\right. \text { and } \\
\left.\left(s_{1} \bmod m, \ldots, s_{k} \bmod m\right) \in A\right\} .
\end{gathered}
$$

In this work we prove that $\mathscr{C}(m, A)$ is a Frobenius pseudo-variety. We also show algorithms that allows us to establish whether a numerical semigroup belongs to $\mathscr{C}(m, A)$ and to compute all the elements of $\mathscr{C}(m, A)$ with a fixed genus. Moreover, we introduce and study three families of numerical semigroups, called of second-level, thin and strong, and corresponding to $\mathscr{C}(m, A)$ when $A=\{1, \ldots, m-1\}^{3}, A=\{(1,1), \ldots,(m-1, m-1)\}$, and $A=$ $\{1, \ldots, m-1\}^{2} \backslash\{(1,1), \ldots,(m-1, m-1)\}$, respectively.

Keywords Modular pseudo-varieties · Second-level numerical semigroups · Thin numerical semigroups $\cdot$ Strong numerical semigroups $\cdot$ Tree associated (with a modular pseudo-variety)

\section{Mathematics Subject Classification 20M14}

\section{Introduction}

Let $\mathbb{N}$ be the set of non-negative integers. A numerical semigroup is a subset $S$ of $\mathbb{N}$ that is closed under addition, $0 \in S$ and $\mathbb{N} \backslash S$ is finite. The Frobenius number of $S$, denoted by

A. M. Robles-Pérez, J. C. Rosales: Both authors are supported by the project MTM2017-84890-P (funded by Ministerio de Economía, Industria y Competitividad and Fondo Europeo de Desarrollo Regional FEDER) and by the Junta de Andalucía Grant Number FQM-343.

$\bowtie$ Aureliano M. Robles-Pérez

arobles@ugr.es

José Carlos Rosales

jrosales@ugr.es

1 Departamento de Matemática Aplicada \& Instituto de Matemáticas (IMAG), Universidad de Granada, 18071 Granada, Spain

2 Departamento de Álgebra \& Instituto de Matemáticas (IMAG), Universidad de Granada, 18071 Granada, Spain 
$\mathrm{F}(S)$, is the greatest integer that does not belong to $S$. The cardinality of $\mathbb{N} \backslash S$, denoted by $\mathrm{g}(S)$, is the genus of $S$.

A Frobenius pseudo-variety is a non-empty family $\mathcal{P}$ of numerical semigroups that fulfils the following conditions.

1. $\mathcal{P}$ has a maximum element (with respect to the inclusion order).

2. If $S, T \in \mathcal{P}$, then $S \cap T \in \mathcal{P}$.

3. If $S \in \mathcal{P}$ and $S \neq \max (\mathcal{P})$, then $S \cup\{\mathrm{F}(S)\} \in \mathcal{P}$.

The multiplicity of a numerical semigroup $S$, denoted by $\mathrm{m}(S)$, is the least positive integer that belongs to $S$. If $m$ is a positive integer, then we denote by $\mathscr{S}_{m}=$ $\{S \mid S$ is a numerical semigroup with $\mathrm{m}(S)=m\}$.

Let $m \in \mathbb{N} \backslash\{0,1\}$ and let $A$ be a finite subset of $\bigcup_{k \in \mathbb{N} \backslash\{0,1\}}\{1, \ldots, m-1\}^{k}$ (where $\left.X^{k}=X \times{ }^{(k)} \cdot \times X=\left\{\left(x_{1}, \ldots, x_{k}\right) \mid x_{1}, \ldots, x_{k} \in X\right\}\right)$. We denote by

$$
\begin{gathered}
\mathscr{C}(m, A)=\left\{S \in \mathscr{S}_{m} \mid s_{1}+\cdots+s_{k}-m \in S \text { if }\left(s_{1}, \ldots, s_{k}\right) \in S^{k}\right. \text { and } \\
\left.\left(s_{1} \bmod m, \ldots, s_{k} \bmod m\right) \in A\right\} .
\end{gathered}
$$

Our main purpose in this work is to study the set $\mathscr{C}(m, A)$.

In Sect. 2 we show that $\mathscr{C}(m, A)$ is a Frobenius pseudo-variety with maximum element given by $\Delta(m)=\{0, m, \rightarrow\}$ (where the symbol $\rightarrow$ means that every integer greater than $m$ belongs to $\Delta(m))$. Thus, we call the pseudo-varieties that arise in this way modular Frobenius pseudo-varieties. Also, we give an algorithm that allows us to establish whether a numerical semigroup belongs to $\mathscr{C}(m, A)$. In addition, with the help of the results in [9], we can arrange $\mathscr{C}(m, A)$ in a rooted tree and we find an algorithm to compute all the elements of $\mathscr{C}(m, A)$ with a fixed genus.

If $X$ is a non-empty subset of $\mathbb{N}$, then we denote by $\langle X\rangle$ the submonoid of $(\mathbb{N},+)$ generated by $X$, that is,

$$
\langle X\rangle=\left\{\lambda_{1} x_{1}+\cdots+\lambda_{n} x_{n} \mid n \in \mathbb{N} \backslash\{0\}, x_{1}, \ldots, x_{n} \in X, \lambda_{1}, \ldots, \lambda_{n} \in \mathbb{N}\right\} .
$$

It is well known (see Lemma 2.1 of [11]) that $\langle X\rangle$ is a numerical semigroup if and only if $\operatorname{gcd}(X)=1$. If $M$ is a submonoid of $(\mathbb{N},+)$ and $M=\langle X\rangle$, then we say that $X$ is a system of generators of $M$. Moreover, if $M \neq\langle Y\rangle$ for any subset $Y \subsetneq X$, then we say that $X$ is a minimal system of generators of $M$. In Corollary 2.8 of [11] it is shown that each submonoid of $(\mathbb{N},+)$ has a unique minimal system of generators and that such a system is finite. We denote by $\operatorname{msg}(M)$ the minimal system of generators of $M$. The cardinality of $\operatorname{msg}(M)$, denoted by e $(M)$, is the embedding dimension of $M$.

By applying Proposition 2.10 of [11], if $S$ is a numerical semigroup, then we know that $\mathrm{e}(S) \leq \mathrm{m}(S)$. A numerical semigroup $S$ has maximal embedding dimension if e $(S)=\mathrm{m}(S)$. This family of numerical semigroups has been extensively studied (for instance, see [2] and [11]). Let us denote by $\mathcal{M}_{m}$ the set formed by the numerical semigroups that have maximal embedding dimension and with multiplicity $m$. It is easy to see that $\mathcal{M}_{m}=\mathscr{C}(m,\{1, \ldots, m-$ $\left.1\}^{2}\right)$.

In Sects. 3, 4, and 5 we study the family $\mathscr{C}(m, A)$ for

- $A=\{1, \ldots, m-1\}^{3}$,

- $A=\{(1,1), \ldots,(m-1, m-1)\}$,

- $A=\{1, \ldots, m-1\}^{2} \backslash\{(1,1), \ldots,(m-1, m-1)\}$,

respectively. Observe that, in a certain sense, these families are generalisation of $\mathcal{M}_{m}$. 
To finish this introduction, we are going to comment several ideas (see $[3,4,14])$ that motivate the study of modular Frobenius pseudo-varieties.

First of all, observe that modular Frobenius pseudo-varieties are related to the nonhomogeneous patterns with positive coefficients that involve in their constant parameter the multiplicity of the numerical semigroup (see [4]).

The notion of non-homogeneous pattern was introduced in [4] as a generalisation of the notion of homogeneous pattern [3]. Thus, a linear pattern $p\left(x_{1}, \ldots, x_{n}\right)$ is an expression of the form $a_{1} x_{1}+\cdots+a_{n} x_{n}+a_{0}$, with $a_{0} \in \mathbb{Z}$ (as usual, $\mathbb{Z}$ is the set of integers numbers) and $a_{1}, \ldots, a_{n} \in \mathbb{Z} \backslash\{0\}$. In particular, the (linear) pattern $p$ is homogeneous if $a_{0}=0$, and non-homogeneous if $a_{0} \neq 0$.

On the other hand, it is said that a numerical semigroup $S$ admits the homogeneous pattern $p$ if $p\left(s_{1}, \ldots, s_{n}\right) \in S$ for every non-increasing sequence $\left(s_{1}, \ldots, s_{n}\right) \in S^{n}$. The corresponding definition for non-homogeneous patterns is a bit different: a numerical semigroup $S$ admits the non-homogeneous pattern $p$ if $p\left(s_{1}, \ldots, s_{n}\right) \in S$ for every non-increasing sequence $\left(s_{1}, \ldots, s_{n}\right) \in(S \backslash\{0\})^{n}$.

Having in mind that $M(S)=S \backslash\{0\}$ is an ideal of the numerical semigroup $S$ (in fact, $M(S)$ is the maximal ideal of $S$ ), in [14] the concepts of the above paragraph were extended in the following way: an ideal $I$ of a numerical semigroups $S$ admits the pattern $p$ if $p\left(s_{1}, \ldots, s_{n}\right) \in S$ for every non-increasing sequence $\left(s_{1}, \ldots, s_{n}\right) \in I^{n}$.

At this point we have the main difference between the above-mentioned papers and our proposal in this work: we do not keep the non-increasing condition (on the sequences in which we evaluate the pattern) in mind. In addition, we take sequences in sets $A$ without structure (that is, $A$ does not have to be an ideal of a numerical semigroup).

Now, let us denote by $\mathscr{S}_{m}(p)$ the family of numerical semigroups with multiplicity $m$ that admit the pattern $p$. If we take the patterns $p_{1}=2 x_{1}+x_{2}-m$ and $p_{2}=x_{1}+2 x_{2}-m$, then $\mathscr{S}_{m}\left(p_{1}, p_{2}\right)=\mathscr{S}_{m}\left(p_{1}\right) \cap \mathscr{S}_{m}\left(p_{2}\right)$ (that is, the family of numerical semigroups which admit $p_{1}$ and $p_{2}$ simultaneously) is equal to $\mathscr{C}(m, A)$ for

$$
A=\left\{\left(a_{1}, a_{2}, a_{3}\right) \in\{1, \ldots, m-1\}^{3} \mid a_{1} \equiv a_{2} \quad(\bmod m)\right\},
$$

being this one an easy example of the connection between modular Frobenius pseudo-varieties and families of numerical semigroups defined by non-homogeneous patterns (as considered in [4]).

A first question studied in $[4,14]$ is the next one: if $p=a_{1} x_{1}+\cdots+a_{n} x_{n}+a_{0}$ is a nonhomogeneous pattern, for which values of $a_{0}$ we have that the family $\mathscr{S}(p)$, of numerical semigroups that admit the pattern $p$, is non-empty? If the answer is affirmative, then it is said that $p$ is an admissible pattern. In our case, we have imposed that $a_{0}=-m$, where $m$ will be the multiplicity of all the numerical semigroups, in order to get a relevant advantage: we want to build in a (more or less) easy and explicit way the Apéry set of the numerical semigroups belonging to $\mathscr{C}(m, A)$. As a consequence of this fact, we will be able to obtain extra information about such families of numerical semigroups.

Of course, in addition to $-m$, it is possible to consider other values that maintain the pseudo-variety structure. Thus, for $p=x_{1}+\cdots+x_{n}+a_{0}$, we have that, independently of the chosen set $A$, every numerical semigroup $S$, such that $a_{0} \in S$, admits the pattern $p$.

Now, let us observe that, if $A_{1} \subseteq A_{2}$, then $\mathscr{C}\left(m, A_{2}\right) \subseteq \mathscr{C}\left(m, A_{1}\right)$. This fact allow us to have sufficient conditions on the patterns $p=a_{1} x_{1}+\cdots+a_{n} x_{n}-k m$, with $k \in \mathbb{N} \backslash\{0,1\}$, from the results in $[4,14]$. For example, from [4, Theorem 4.1] or [14, Proposition 20], we can assert that $\mathscr{C}(m, A) \neq \emptyset$ if $n-k \geq 1$.

A second question that appears in $[3,14]$ is about the equivalence of patterns. Briefly, the pattern $p_{1}$ induces the pattern $p_{2}$ if $\mathscr{S}\left(p_{2}\right) \subseteq \mathscr{S}\left(p_{1}\right)$ and, moreover, $p_{1}$ and $p_{2}$ are equivalent 
patterns if they induce each other. Maybe the first result of this type is the equivalence between the homogeneous patterns $2 x_{1}-x_{2}$ and $x_{1}+x_{2}-x_{3}$, which correspond to the family of Arf numerical semigroups (see [5]). Since the set $A$ has an important role (not to say the main role) in the families $\mathscr{C}(m, A)$, the large number of possibilities in the choice of $A$ leads us to believe that this is an issue that deserves a new work.

By the way, in Sect. 4 we study the family of thin numerical semigroups, denoted by $\mathscr{T}_{m}$, and in Sect. 5 we consider the family of strong numerical semigroups, denoted by $\mathscr{R}_{m}$. These families are associated with the patterns $2 x-m$ and $x+y-m$, respectively. However, the set $A$ is different in each case and, as a consequence, we have that there is not a inclusion relation between both of them (see Examples 4.6 and 5.6). This fact may give an idea about the difficulty of obtaining similar results to those seen in $[3,14]$.

In any case, we can show simple results about the equivalence (or, at least, the inclusions) of the families $\mathscr{C}(m, A)$.

Remark 1.1 All the patterns $p=x_{1}+\cdots+x_{n}-m$ are equivalent (independently of the chosen set $A$ ) if $n \geq m$, in which case we have that $\mathscr{C}(m, A)=\mathscr{S}_{m}$. Indeed, applying the pigeonhole principle, if $s_{1}, \ldots, s_{n}$ are elements of $S \in \mathscr{S}_{m}$ such that $s_{i} \not \equiv 0(\bmod m)$, $1 \leq i \leq n$, then there exist $i, j \in\{1, \ldots, n\}$, with $i<j$, such that $s_{i}+\cdots+s_{j}=k m$ for some $k \in \mathbb{N} \backslash\{0\}$. Consequently, $S \in \mathscr{C}(m, A)$.

Remark 1.2 Let us set $A=\{(1,1),(3,4)\}, B=\{(1,1,2),(1,3,1),(1,3,4)\}$, and $m \geq 5$. Then $\mathscr{C}(m, A) \subseteq \mathscr{C}(m, B)$. In order to verify this inclusion, we take $a_{1}, a_{2}, a_{3} \in S \in$ $\mathscr{C}(m, A)$.

- If $a_{1} \equiv 1(\bmod m), a_{2} \equiv 1(\bmod m), a_{3} \equiv 2(\bmod m)$, then $a_{1}+a_{2}+a_{3}-m=$ $\left(a_{1}+a_{2}-m\right)+a_{3} \in S$.

- If $a_{1} \equiv 1(\bmod m), a_{2} \equiv 3(\bmod m), a_{3} \equiv 1(\bmod m)$, then $a_{1}+a_{2}+a_{3}-m=$ $\left(a_{1}+a_{3}-m\right)+a_{2} \in S$.

- If $a_{1} \equiv 1(\bmod m), a_{2} \equiv 3(\bmod m), a_{3} \equiv 4(\bmod m)$, then $a_{1}+a_{2}+a_{3}-m=$ $\left(a_{2}+a_{3}-m\right)+a_{1} \in S$.

More generally, let us suppose that, for each $b \in B$, there exists $a \in A$ such that $b$ is obtained by adding coordinates to $a$. Then $\mathscr{C}(m, A) \subseteq \mathscr{C}(m, B)$.

As a final recommendation, it is worth mentioning that in [4, Section 2] and in [14, Introduction] there are several motivating examples, and references, as to why it is interesting to study (non-homogeneous) patterns of numerical semigroups.

\section{The pseudo-variety $\mathscr{C}(\boldsymbol{m}, \boldsymbol{A})$}

In this section, $m$ is an integer greater than or equal to 2 and $A$ is a finite subset of $\bigcup_{k \in \mathbb{N} \backslash\{0,1\}}\{1, \ldots, m-1\}^{k}$. Moreover, recall that

$$
\begin{gathered}
\mathscr{C}(m, A)=\left\{S \in \mathscr{S}_{m} \mid s_{1}+\cdots+s_{k}-m \in S \text { if }\left(s_{1}, \ldots, s_{k}\right) \in S^{k}\right. \text { and } \\
\left.\left(s_{1} \bmod m, \ldots, s_{k} \bmod m\right) \in A\right\},
\end{gathered}
$$

where $x$ mod $m$ denotes the remainder after division of $x$ by $m$.

If $S$ is a numerical semigroup and $x \in S \backslash\{0\}$, then the Apéry set of $x$ in $S$ (see [1]) is $\operatorname{Ap}(S, x)=\{w(0)=0, w(1), \ldots, w(x-1)\}$, where $w(i)$ is the least element of $S$ that is congruent with $i$ modulus $x$. Observe that an integer $s$ belongs to $S$ if and only if there exists $t \in \mathbb{N}$ such that $s=w(s \bmod x)+t x$. 
Proposition 2.1 Let $S \in \mathscr{S}_{m}$ and $\operatorname{Ap}(S, m)=\{w(0)=0, w(1), \ldots, w(m-1)\}$. Then the following conditions are equivalent.

1. $S \in \mathscr{C}(m, A)$.

2. $w\left(i_{1}\right)+\cdots+w\left(i_{k}\right)-m \in S$ for all $\left(i_{1}, \ldots, i_{k}\right) \in A$.

Proof $\left(1 . \Rightarrow 2\right.$.) Since $w\left(i_{1}\right), \ldots, w\left(i_{k}\right) \in S$ and $\left(w\left(i_{1}\right) \bmod m, \ldots, w\left(i_{k}\right) \bmod m\right)=$ $\left(i_{1}, \ldots, i_{k}\right) \in A$, then $w\left(i_{1}\right)+\cdots+w\left(i_{k}\right)-m \in S$.

(2. $\Rightarrow$ 1.) Let $s_{1}, \ldots, s_{k} \in S$ such that

$$
\left(s_{1} \bmod m, \ldots, s_{k} \bmod m\right)=\left(i_{1}, \ldots, i_{k}\right) \in A .
$$

Then there exist $t_{1}, \ldots, t_{k} \in \mathbb{N}$ such that $s_{j}=w\left(i_{j}\right)+t_{j} m, 1 \leq j \leq k$, and thus, $s_{1}+\cdots+$ $s_{k}=\left(w\left(i_{1}\right)+\cdots+w\left(i_{k}\right)-m\right)+\left(t_{1}+\cdots+t_{k}\right) m \in S$.

By using the function AperyListofNumericalSemigroupWRTElement $(\mathrm{S}, \mathrm{m})$ of [6], we can compute $\operatorname{Ap}(S, m)$ from a system of generators of $S$. Thereby, we have the following algorithm to decide if a numerical semigroup $S$ belongs or not to $\mathscr{C}(m, A)$.

Algorithm 2.2 INPUT: A finite subset $G$ of positive integers.

OUTPUT: $\langle G\rangle \in \mathscr{C}(m, A)$ or $\langle G\rangle \notin \mathscr{C}(m, A)$.

(1) If $\min (\mathrm{G}) \neq m$, return $\langle G\rangle \notin \mathscr{C}(m, A)$.

(2) If $\operatorname{gcd}(G) \neq 1$, return $\langle G\rangle \notin \mathscr{C}(m, A)$.

(3) Compute $\operatorname{Ap}(\langle G\rangle, m)=\{w(0), w(1), \ldots, w(m-1)\}$.

(4) If $w\left(i_{1}\right)+\cdots+w\left(i_{k}\right)-m \in S$ for all $\left(i_{1}, \ldots, i_{k}\right) \in A$, return $\langle G\rangle \in \mathscr{C}(m, A)$.

(5) Return $\langle G\rangle \notin \mathscr{C}(m, A)$.

Let us illustrate the working of the previous algorithm through an example.

Example 2.3 Let us make use of Algorithm 2.2 with $G=\{5,7,9\}, m=5$, and $A=$ $\{(1,3),(2,2)\}$.

- $\min (G)=5$.

- $\operatorname{gcd}(G)=1$.

- $\operatorname{Ap}(\langle G\rangle, 5)=\{w(0)=0, w(1)=16, w(2)=7, w(3)=18, w(4)=19\}$.

- $w(1)+w(3)-5=29 \in\langle G\rangle$ and $w(2)+w(2)-5=9 \in\langle G\rangle$.

Therefore, $\langle G\rangle=\langle 5,7,9\rangle \in \mathscr{C}(5,\{(1,3),(2,2)\})$.

Recall that, if $m$ is an integer greater than or equal to 2, then we denote by $\Delta(m)=$ $\{0, m, \rightarrow\}$. It is clear that $\Delta(m) \in \mathscr{S}_{m}$ and that, if $s_{1}, \ldots, s_{k} \in \Delta(m) \backslash\{0\}$ and $k \geq 2$, then $s_{1}+\cdots+s_{k}-m \in \Delta(m)$. Therefore, we have the next result.

Lemma 2.4 Let $m \in \mathbb{N} \backslash\{0,1\}$ and $A \subseteq \bigcup_{k \in\{2, \rightarrow\}}\{1, \ldots, m-1\}^{k}$ (A finite). Then $\Delta(m) \in$ $\mathscr{C}(m, A)$.

Let $m \in \mathbb{N} \backslash\{0,1\}$. Then it is easy to show that $S \cap T \in \mathscr{S}_{m}$ for all $S, T \in \mathscr{S}_{m}$. Moreover, $\Delta(m)$ is the maximum of $\mathscr{S}_{m}$ and, if $S \in \mathscr{S}_{m}$ and $S \neq \Delta(m)$, then $S \cup\{\mathrm{F}(S)\} \in \mathscr{S}_{m}$. From all this, we conclude the following result.

Lemma 2.5 Let $m \in \mathbb{N} \backslash\{0,1\}$. Then $\mathscr{S}_{m}$ is a Frobenius pseudo-variety with $\Delta(m)$ as maximum element.

Proposition 2.6 $\mathscr{C}(m, A)$ is a Frobenius pseudo-variety. 
Proof From Lemmas 2.4 and 2.5, we have that $\Delta(m)$ is the maximum of $\mathscr{C}(m, A)$. It is also easy to see that, if $S, T \in \mathscr{C}(m, A)$, then $S \cap T \in \mathscr{C}(m, A)$. In order to finish the proof, let us see that, if $S \in \mathscr{C}(m, A)$ and $S \neq \Delta(m)$, then $S \cup\{\mathrm{F}(S)\} \in \mathscr{C}(m, A)$. For that, we have to show that, if $s_{1}, \ldots, s_{k} \in S \cup\{\mathrm{F}(S)\}$ and $\left(s_{1} \bmod m, \ldots, s_{k} \bmod m\right) \in A$, then $s_{1}+\cdots+s_{k}-m \in S \cup\{\mathrm{F}(S)\}$. In effect, if $\mathrm{F}(S) \notin\left\{s_{1}, \ldots, s_{k}\right\}$, then the result is true because $S \in \mathscr{C}(m, A)$. On the other hand, if $\mathrm{F}(S) \in\left\{s_{1}, \ldots, s_{k}\right\}$, then $s_{1}+\cdots+s_{k}-m \geq \mathrm{F}(S)$ and, consequently, $s_{1}+\cdots+s_{k}-m \in S \cup\{\mathrm{F}(S)\}$.

Our next purpose in this section is to show an algorithm that allows us to build all the elements of $\mathscr{C}(m, A)$ that have a fixed genus. To do that, we use the concept of rooted tree.

A graph $G$ is a pair $(V, E)$ where $V$ is a non-empty set (whose elements are called vertices of $G$ ) and $E$ is a subset of $\{(v, w) \in V \times V \mid v \neq w\}$ (whose elements are called edges of $G$ ). A path (of length $n$ ) connecting the vertices $x$ and $y$ of $G$ is a sequence of different edges $\left(v_{0}, v_{1}\right),\left(v_{1}, v_{2}\right), \ldots,\left(v_{n-1}, v_{n}\right)$ such that $v_{0}=x$ and $v_{n}=y$.

We say that a graph $G$ is a (rooted) tree if there exists a vertex $r$ (known as the root of $G$ ) such that, for any other vertex $x$ of $G$, there exists a unique path connecting $x$ and $r$. If there exists a path connecting $x$ and $y$, then we say that $x$ is a descendant of $y$. In particular, if $(x, y)$ is an edge of the tree, then we say that $x$ is a child of $y$. (See [12].)

We define the graph $\mathrm{G}(\mathscr{C}(m, A))$ in the following way: $\mathscr{C}(m, A)$ is the set of vertices and $\left(S, S^{\prime}\right) \in \mathscr{C}(m, A) \times \mathscr{C}(m, A)$ is an edge if $S \cup\{\mathrm{F}(S)\}=S^{\prime}$. The following result is a consequence of Lemma 4.2 and Theorem 4.3 of [9].

Theorem $2.7 \mathrm{G}(\mathscr{C}(m, A))$ is a tree with root $\Delta(m)$. Moreover, the children set of $S \in$ $\mathscr{C}(m, A)$ is $\{S \backslash\{x\} \mid x \in \operatorname{msg}(S), S \backslash\{x\} \in \mathscr{C}(m, A), x>\mathrm{F}(S)\}$.

Let $S$ be a numerical semigroup and let $x \in S$. Then it is clear that $S \backslash\{x\}$ is a numerical semigroup if and only if $x \in \operatorname{msg}(S)$. Moreover, let us observe that, if $x \in \operatorname{msg}(S)$ and $m \in S \backslash\{0, x\}$, then

$$
\operatorname{Ap}(S \backslash\{x\}, m)=(\operatorname{Ap}(S, m) \backslash\{x\}) \cup\{x+m\} .
$$

In the following result we characterise the children of $S \in \mathscr{C}(m, A)$.

Proposition 2.8 Let $S \in \mathscr{C}(m, A), \operatorname{Ap}(S, m)=\{w(0), w(1), \ldots, w(m-1)\}$ and $x \in$ $\operatorname{msg}(S) \backslash\{m\}$. Then $S \backslash\{x\} \in \mathscr{C}(m, A)$ if and only if $w\left(i_{1}\right)+\cdots+w\left(i_{k}\right) \neq m+x$ for all $\left(i_{1}, \ldots, i_{k}\right) \in A$.

Proof Let us suppose that $\operatorname{Ap}(S \backslash\{x\}, m)=(\operatorname{Ap}(S, m) \backslash\{x\}) \cup\{x+m\}=\left\{w^{\prime}(0), w^{\prime}(1), \ldots\right.$, $\left.w^{\prime}(m-1)\right\}$.

(Necessity.) If $w\left(i_{1}\right)+\cdots+w\left(i_{k}\right)=m+x$, then $m+x \notin\left\{w\left(i_{1}\right), \ldots, w\left(i_{k}\right)\right\}$ and, therefore, $w\left(i_{1}\right)=w^{\prime}\left(i_{1}\right), \ldots, w\left(i_{k}\right)=w^{\prime}\left(i_{k}\right)$. Moreover, $w\left(i_{1}\right)+\cdots+w\left(i_{k}\right)-m=x \notin S \backslash\{x\}$ and, consequently, $S \backslash\{x\} \notin \mathscr{C}(m, A)$.

(Sufficiency.) In order to prove that $S \backslash\{x\} \in \mathscr{C}(m, A)$, by Proposition 2.1, it is enough to see that, if $\left(i_{1}, \ldots, i_{k}\right) \in A$, then $w^{\prime}\left(i_{1}\right)+\cdots+w^{\prime}\left(i_{k}\right)-m \in S \backslash\{x\}$.

Since $S \in \mathscr{C}(m, A)$, we easily deduce that $w^{\prime}\left(i_{1}\right)+\cdots+w^{\prime}\left(i_{k}\right)-m \in S$. Now, if $w^{\prime}\left(i_{1}\right)+\cdots+w^{\prime}\left(i_{k}\right)-m \notin S \backslash\{x\}$, then $w^{\prime}\left(i_{1}\right)+\cdots+w^{\prime}\left(i_{k}\right)=m+x$. Thus, $w\left(i_{1}\right)=$ $w^{\prime}\left(i_{1}\right), \ldots, w\left(i_{k}\right)=w^{\prime}\left(i_{k}\right)$ and $w\left(i_{1}\right)+\cdots+w\left(i_{k}\right)=m+x$, where the last equality is in contradiction with $S \in \mathscr{C}(m, A)$.

Let us observe that a tree can be built in a recurrent way starting from its root and connecting each vertex with its children. Let us also observe that the elements of $\mathscr{C}(m, A)$ with genus equal to $g+1$ are precisely the children of the elements of $\mathscr{C}(m, A)$ with genus equal to $g$.

We are ready to show the above announced algorithm. 
Algorithm 2.9 INPUT: A positive integer $g$.

OUTPUT: $\{S \in \mathscr{C}(m, A) \mid \mathrm{g}(S)=g\}$.

(1) If $g<m-1$, return $\emptyset$.

(2) $X=\{\Delta(m)\}$ and $i=m-1$.

(3) If $i=g$, return $X$.

(4) For each $S \in X$, compute the set

$$
\mathscr{B}_{S}=\{x \in \operatorname{msg}(S) \mid x>\mathrm{F}(S), x \neq m, S \backslash\{x\} \in \mathscr{C}(m, A)\} .
$$

(5) If $\bigcup_{S \in X} \mathscr{B}_{S}=\emptyset$, return $\emptyset$.

(6) $X=\bigcup_{S \in X}\left\{S \backslash\{x\} \mid x \in \mathscr{B}_{S}\right\}, i=i+1$, and go to (3).

For computing item (4) in the above algorithm, we use Proposition 2.8 and the following Lemma 2.10, which is a reformulation of Corollary 18 of [8], and is useful to obtain the minimal system of generators of $S \backslash\{x\}$ starting from the minimal system of generators of $S$.

Lemma 2.10 Let $S$ be a numerical semigroup with $\operatorname{msg}(S)=\left\{n_{1}<n_{2}<\cdots<n_{e}\right\}$. If $i \in\{2, \ldots, e\}$ and $n_{i}>\mathrm{F}(S)$, then

$$
\operatorname{msg}\left(S \backslash\left\{n_{i}\right\}\right)=\left\{\begin{array}{r}
\left\{n_{1}, \ldots, n_{e}\right\} \backslash\left\{n_{i}\right\}, \quad \text { if there exists } j \in\{2, \ldots, i-1\} \\
\text { such that } n_{i}+n_{1}-n_{j} \in S ; \\
\left(\left\{n_{1}, \ldots, n_{e}\right\} \backslash\left\{n_{i}\right\}\right) \cup\left\{n_{i}+n_{1}\right\}, \quad \text { in other case. }
\end{array}\right.
$$

We illustrate the functioning of Algorithm 2.9 with an example.

Example 2.11 Let us compute all the elements of $\mathscr{C}(5,\{(1,1),(1,2)\})$ with genus equal to 6.

- $X=\{\langle 5,6,7,8,9\rangle\}$ and $i=4$.

- $B_{\langle 5,6,7,8,9\rangle}=\{6,9\}$.

- $X=\{\langle 5,7,8,9,11\rangle,\langle 5,6,7,8\rangle\}$ and $i=5$.

- $B_{\langle 5,7,8,9,11\rangle}=\{7,8,9,11\}$ and $B_{\langle 5,6,7,8\rangle}=\emptyset$.

- $X=\{\langle 5,8,9,11,12\rangle,\langle 5,7,9,11,13\rangle,\langle 5,7,8,11\rangle,\langle 5,7,8,9\rangle\}$ and $i=6$.

- Return $\{\langle 5,8,9,11,12\rangle,\langle 5,7,9,11,13\rangle,\langle 5,7,8,11\rangle,\langle 5,7,8,9\rangle\}$.

Taking advantage of the above example, we finish this section building the first three levels of the tree $\mathrm{G}(\mathscr{C}(5,\{(1,1),(1,2)\}))$.

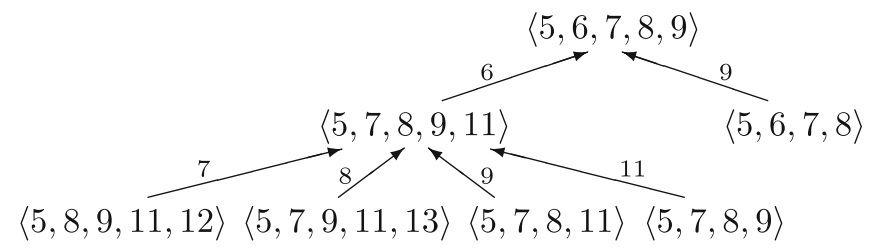

Observe that the number which appear next to each edge $\left(S^{\prime}, S\right)$ is the minimal generator $x$ of $S$ such that $S^{\prime}=S \backslash\{x\}$.

\section{Second-level numerical semigroups}

We say that a numerical semigroup $S$ is of second-level if $x+y+z-\mathrm{m}(S) \in S$ for all $(x, y, z) \in(S \backslash\{0\})^{3}$. We denote by $\mathscr{L}_{2, m}$ the set of all the second-level numerical semigroups with multiplicity equal to $m$. 
Proposition 3.1 Let $S$ be a numerical semigroup with minimal system of generators given by $\left\{m=n_{1}<n_{2}<\cdots<n_{e}\right\}$. Then the following two conditions are equivalents.

1. $S \in \mathscr{L}_{2, m}$.

2. If $(i, j, k) \in\{2, \ldots, e\}^{3}$, then $n_{i}+n_{j}+n_{k}-m \in S$.

Proof $(1 . \Rightarrow 2$.) It is evident from the definition of second-level numerical semigroup.

$\left(2 . \Rightarrow 1\right.$.) Let $(x, y, z) \in(S \backslash\{0\})^{3}$. If $0 \in\{x \bmod m, y \bmod m, z \bmod m\}$, then it is clear that $x+y+z-m \in S$. Now, if $0 \notin\{x \bmod m, y \bmod m, z \bmod m\}$, then we easily deduce that there exist $(i, j, k) \in\{2, \ldots, e\}^{3}$ and $\left(s_{1}, s_{2}, s_{3}\right) \in S^{3}$ such that $(x, y, z)=$ $\left(n_{i}, n_{j}, n_{k}\right)+\left(s_{1}, s_{2}, s_{3}\right)$. Therefore, $x+y+z-m=\left(n_{i}+n_{j}+n_{k}-m\right)+s_{1}+s_{2}+s_{3} \in S$. Consequently, $S \in \mathscr{L}_{2, m}$.

The above proposition allows us to easily decide whether a numerical semigroup is of second-level or not.

Example 3.2 Let us see that $S=\langle 5,7,16\rangle \in \mathscr{L}_{2, m}$. In effect, it is clear that $\{7+7+7-$ $5,7+7+16-5,7+16+16,16+16+16-5\}=\{16,25,34,43\} \subseteq S$. Therefore, by Proposition 3.1, we have that $S \in \mathscr{L}_{2, m}$.

Now our intention is to show that $\mathscr{L}_{2, m}$ is a modular Frobenius pseudo-variety.

Proposition 3.3 Let $m \in \mathbb{N} \backslash\{0,1\}$. Then $\mathscr{L}_{2, m}=\mathscr{C}\left(m,\{1, \ldots, m-1\}^{3}\right)$.

Proof Let $S \in \mathscr{L}_{2, m}$ and $\operatorname{Ap}(S, m)=\{w(0), w(1), \ldots, w(m-1)\}$. If $(i, j, k) \in\{1, \ldots, m-$ $1\}^{3}$, then $(w(i), w(j), w(k)) \in(S \backslash\{0\})^{3}$ and, therefore, $w(i)+w(j)+w(k)-m \in S$. By applying Proposition 2.1, we have that $S \in \mathscr{C}\left(m,\{1, \ldots, m-1\}^{3}\right)$.

In order to see the other inclusion, let $S \in \mathscr{C}\left(m,\{1, \ldots, m-1\}^{3}\right)$ and $(x, y, z) \in(S \backslash\{0\})^{3}$. Firstly, if $0 \in\{x \bmod m, y \bmod m, z \bmod m\}$, then it is clear that $x+y+z-m \in S$. Secondly, if $0 \notin\{x \bmod m, y \bmod m, z \bmod m\}$, then there exist $(i, j, k) \in\{1, \ldots, m-1\}^{3}$ and $(p, q, r) \in \mathbb{N}^{3}$ such that $x=w(i)+p m, y=w(j)+q m$, and $z=w(k)+r m$. Thus, $x+y+z-m=(w(i)+w(j)+w(k)-m)+(p+q+r) m \in S$ and, therefore, $S \in \mathscr{L}_{2, m}$.

As an immediate consequence of Propositions 2.6 and 3.3, we have the following result.

Corollary 3.4 Let $m \in \mathbb{N} \backslash\{0,1\}$. Then $\mathscr{L}_{2, m}$ is a modular pseudo-Frobenius variety and, in addition, $\Delta(m)$ is the maximum of $\mathscr{L}_{2, m}$.

Our next step is to build the tree associated with the pseudo-variety $\mathscr{L}_{2, m}$. To do this, we should characterise the possible children of each element in $\mathscr{L}_{2, m}$.

Let $S$ be a numerical semigroup with $\operatorname{msg}(S)=\left\{n_{1}, \ldots, n_{e}\right\}$. If $s \in S$, then we denote by

$$
L_{S}(s)=\max \left\{a_{1}+\cdots+a_{e} \mid\left(a_{1}, \ldots, a_{e}\right) \in \mathbb{N}^{e} \text { and } a_{1} n_{1}+\cdots+a_{e} n_{e}=s\right\} .
$$

Proposition 3.5 Let $m \in \mathbb{N} \backslash\{0,1\}, S \in \mathscr{L}_{2, m}$, and $x \in \operatorname{msg}(S) \backslash\{m\}$. Then $S \backslash\{x\} \in \mathscr{L}_{2, m}$ if and only if $L_{S \backslash\{x\}}(x+m) \leq 2$.

Proof (Necessity.) Let us suppose that $L_{S \backslash\{x\}}(x+m) \geq 3$. Then there exists $(a, b, c) \in$ $(S \backslash\{0, x\})^{3}$ such that $x+m=a+b+c$. Thus, $a+b+c-m=x \notin S \backslash\{x\}$ and, therefore, $S \backslash\{x\} \notin \mathscr{L}_{2, m}$.

(Sufficiency.) If $(a, b, c) \in(S \backslash\{0, x\})^{3}$, then $a+b+c-m \in S$, since $S \in \mathscr{L}_{2, m}$. Now, if $a+b+c-m=x$, then $L_{S \backslash\{x\}}(x+m) \geq 3$. Therefore, $a+b+c-m \neq x$ and, consequently, $a+b+c-m \in S \backslash\{x\}$. Thus, we conclude that $S \backslash\{x\} \in \mathscr{L}_{2, m}$. 
By applying Theorem 2.7, Propositions 3.3 and 3.5, and Lemma 2.10, we can easily build the tree $\mathrm{G}\left(\mathscr{L}_{2, m}\right)$.

Example 3.6 The first four levels of $\mathrm{G}\left(\mathscr{L}_{2,4}\right)$ appear in the following figure.

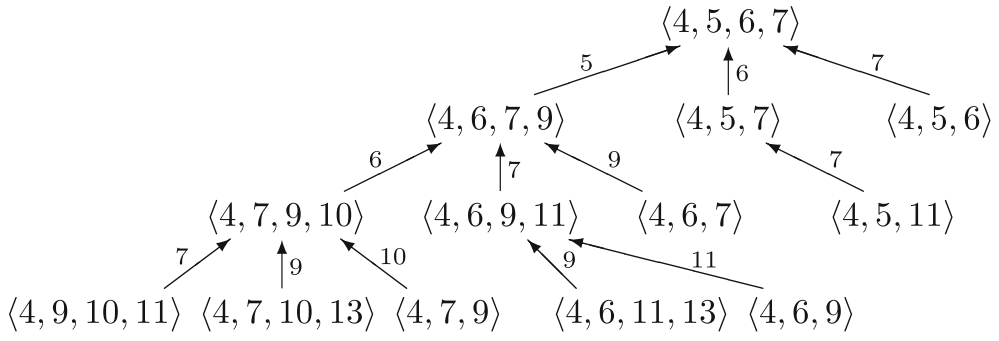

The Frobenius problem (see [7]) consists in finding formulas that allow us to compute the Frobenius number and the genus of a numerical semigroup in terms of the minimal system of generators of such a numerical semigroup. This problem was solved in [15] for numerical semigroups with embedding dimension two. At present, the Frobenius problem is open for embedding dimension greater than or equal to 3. However, if we know the Apéry set $\operatorname{Ap}(S, x)$ for some $x \in S \backslash\{0\}$, then we have solved the Frobenius problem for $S$ because we have the following result from [13].

Lemma 3.7 Let $S$ be a numerical semigroup and let $x \in S \backslash\{0\}$. Then

1. $\mathrm{F}(S)=(\max (\operatorname{Ap}(S, x)))-x$,

2. $\mathrm{g}(S)=\frac{1}{x}\left(\sum_{w \in \operatorname{Ap}(S, x)} w\right)-\frac{x-1}{2}$.

The knowledge of $\operatorname{Ap}(S, x)=\{w(0), w(1), \ldots, w(x-1)\}$ also allows us to determine the membership of an integer to the numerical semigroup $S$. In fact, if $n \in \mathbb{N}$, then $n \in S$ if and only if $n \geq w(n \bmod x)$.

Now our purpose is to show that, if $S \in \mathscr{L}_{2, m}$, then is rather easy to compute $\operatorname{Ap}(S, m)$. We need the following easy result.

Lemma 3.8 Let $m \in \mathbb{N} \backslash\{0,1\}, S \in \mathscr{L}_{2, m}, \operatorname{msg}(S)=\left\{n_{1}=m, n_{2}, \ldots, n_{e}\right\}$. Then $\left\{0, n_{2}, \ldots, n_{e}\right\} \subseteq \operatorname{Ap}(S, m) \subseteq\left\{0, n_{2}, \ldots, n_{e}\right\} \cup\left\{n_{i}+n_{j} \mid(i, j) \in\{2, \ldots, e\}^{2}\right\}$.

As an immediate consequence of the above lemma we can formulate the following result.

Proposition 3.9 Let $m \in \mathbb{N} \backslash\{0,1\}, S \in \mathscr{L}_{2, m}, \operatorname{msg}(S)=\left\{n_{1}=m, n_{2}, \ldots, n_{e}\right\}$. Then $\operatorname{Ap}(S, m)=\{w(0), w(1), \ldots, w(m-1)\}$ where $w(i)$ is the least element of $\left\{0, n_{2}, \ldots, n_{e}\right\} \cup$ $\left\{n_{i}+n_{j} \mid(i, j) \in\{2, \ldots, e\}^{2}\right\}$ that is congruent to $i$ modulo $m$.

Corollary 3.10 Let $m \in \mathbb{N} \backslash\{0,1\}$ and $S \in \mathscr{L}_{2, m}$. Then $m=\mathrm{m}(S) \leq \frac{\mathrm{e}(S)(\mathrm{e}(S)+1)}{2}$.

Following the notation introduced in [10], we say that $x \in \mathbb{Z} \backslash S$ is a pseudo-Frobenius number of $S$ if $x+s \in S$ for all $s \in S \backslash\{0\}$. We denote by $\operatorname{PF}(S)$ the set of all the pseudoFrobenius numbers of $S$. The cardinality of $\operatorname{PF}(S)$ is an important invariant of $S$ (see [2]) that is the so-called type of $S$ and it is denoted by $\mathrm{t}(S)$.

Let $S$ be a numerical semigroup. Then we define the following binary relation over $\mathbb{Z}$ : $a \leq_{S} b$ if $b-a \in S$. In [11] it is shown that $\leq_{S}$ is a partial order (that is, reflexive, transitive, and antisymmetric). Moreover, Proposition 2.20 of [11] is the next result. 
Proposition 3.11 Let $S$ be a numerical semigroup and $x \in S \backslash\{0\}$. Then

$$
\operatorname{PF}(S)=\left\{w-x \mid w \in \text { Maximals }_{\leq S}(\operatorname{Ap}(S, x))\right\}
$$

Let us observe that, if $w, w^{\prime} \in \operatorname{Ap}(S, x)$, then $w^{\prime}-w \in S$ if and only if $w^{\prime}-w \in \operatorname{Ap}(S, x)$. Therefore, Maximals ${ }_{\leq S}(\operatorname{Ap}(S, x))$ is the set

$$
\left\{w \in \operatorname{Ap}(S, x) \mid w^{\prime}-w \notin \operatorname{Ap}(S, x) \backslash\{0\} \text { for all } w^{\prime} \in \operatorname{Ap}(S, x)\right\} .
$$

We finish this section with an example that illustrates the above results.

Example 3.12 Having in mind that $S=\langle 5,6,13\rangle$ is a second-level numerical semigroup, it is easy to compute $\operatorname{Ap}(S, 5)$. In fact, by applying Lemma 3.8, we have that $\operatorname{Ap}(S, 5) \subseteq\{0,6,13\} \cup\{12,19,26\}$ and, by Proposition 3.9, we conclude that $\operatorname{Ap}(S, 5)=$ $\{0,6,12,13,19\}$. On the other hand, by Lemma 3.7, we know that $\mathrm{F}(S)=19-5=14$ and $\mathrm{g}(S)=\frac{1}{5}(6+12+13+19)-\frac{5-1}{2}=8$. Finally, since Maximals ${ }_{\leq S}(\operatorname{Ap}(S, 5))=\{12,19\}$, Proposition 3.11 allows us to claim that $\operatorname{PF}(S)=\{7,12\}$ and $\mathrm{t}(S)=2$.

Remark 3.13 The definition of second-level numerical semigroup can be easily generalised to greater levels. Thus, we say that a numerical semigroup is of $n$ th-level if $x_{1}+\cdots+x_{n+1}-$ $\mathrm{m}(S) \in S$ for all $\left(x_{1}, \ldots, x_{n+1}\right) \in(S \backslash\{0\})^{n+1}$ and denote by $\mathscr{L}_{n, m}$ the set of all the $n$ th-level numerical semigroups with multiplicity equal to $m$.

It is clear that for $n$ th-level we obtain similar results to those of second-level. In particular, $\mathscr{L}_{n, m}=\mathscr{C}\left(m,\{1, \ldots, m-1\}^{n+1}\right)$ and Proposition 3.5 remains true taking $L_{S \backslash\{x\}}(x+m) \leq n$.

On the other hand, having in mind that $\mathscr{L}_{1, m}$ is the family of numerical semigroups with maximal embedding dimension and that (by Remark 1.1) $\mathscr{L}_{m-1, m}=\mathscr{S}_{m}$, we can observe that

$$
\mathscr{L}_{1, m} \subseteq \mathscr{L}_{2, m} \subseteq \cdots \subseteq \mathscr{L}_{m-1, m}=\mathscr{S}_{m},
$$

where all the inclusions are strict, as we can deduce from the following example.

Example 3.14 Let us set $m=5$ and

$$
S_{1}=\langle 5,6\rangle=\{0,5,6,10,11,12,15,16,17,18,20, \rightarrow\} .
$$

Then,

- $S_{1} \in \mathscr{L}_{4,5} \backslash \mathscr{L}_{3,5}=\mathscr{S}_{5} \backslash \mathscr{L}_{3,5}$, because $4 \times 6-5 \notin S_{1}$ and $s_{1}+\cdots+s_{5}-5 \geq 5 \times 6-5 \geq$ $F\left(S_{1}\right)+1$;

- $S_{2}=S_{1} \cup\{19\}=\langle 5,6,19\rangle \in \mathscr{L}_{3,5} \backslash \mathscr{L}_{2,5}$, because $3 \times 6-5 \notin S_{2}$ and $s_{1}+\cdots+s_{4}-5 \geq$ $4 \times 6-5 \geq F\left(S_{2}\right)+1$;

- $S_{3}=S_{2} \cup\{13,14\}=\langle 5,6,13,14\rangle \in \mathscr{L}_{2,5} \backslash \mathscr{L}_{1,5}$, because $2 \times 6-5 \notin S_{2}$ and $s_{1}+s_{2}+s_{3}-5 \geq 3 \times 6-5 \geq F\left(S_{3}\right)+1$.

Generalising this example to other values of $m$ is trivial.

Remark 3.15 Let us observe that the chain obtained in Remark 3.13 is reminiscent, in some sense, of the chain associated with subtraction patterns (see [3, Section 6]).

\section{Thin numerical semigroups}

We say that a numerical semigroup $S$ is thin if $2 x-\mathrm{m}(S) \in S$ for all $x \in S \backslash\{0\}$. We denote by $\mathscr{T}_{m}$ the set of all the thin numerical semigroups with multiplicity equal to $m$. 
Proposition 4.1 Let $S$ be a numerical semigroup with minimal system of generators given by $\left\{m=n_{1}<n_{2}<\cdots<n_{e}\right\}$. Then the following two conditions are equivalents.

1. $S \in \mathscr{T}_{m}$.

2. $2 n_{i}-m \in$ S for all $i \in\{2, \ldots, e\}$.

Proof $(1 . \Rightarrow 2$.) It follows from the definition of thin numerical semigroup.

(2. $\Rightarrow 1$.) Let $x \in S \backslash\{0\}$. If $x \equiv 0(\bmod m)$, then it is clear that $2 x-m \in S$. Now, if $x \not \equiv 0(\bmod m)$, then we have that there exist $i \in\{2, \ldots, e\}$ and $s \in S$ such that $x=n_{i}+s$. Therefore, $2 x-m=\left(2 n_{i}-m\right)+2 s \in S$ and, consequently, $S \in \mathscr{T}_{m}$.

The above proposition allows us to easily decide whether a numerical semigroup is thin or not.

Example 4.2 Let us see that $S=\langle 4,6,7\rangle \in \mathscr{T}_{4}$. In effect, it is clear that $\{2 \cdot 6-4,2 \cdot 7-4\}=$ $\{8,10\} \subseteq S$. Therefore, by Proposition 4.1, we have that $S \in \mathscr{T}_{m}$.

Now we want to show that $\mathscr{T}_{m}$ is a modular Frobenius pseudo-variety.

Proposition $4.3 \mathscr{T}_{m}=\mathscr{C}(m,\{(1,1),(2,2) \ldots,(m-1, m-1)\})$ for all $m \in \mathbb{N} \backslash\{0,1\}$.

Proof If $S \in \mathscr{T}_{m}$ and $\operatorname{Ap}(S, m)=\{w(0), w(1), \ldots, w(m-1)\}$, then it is clear that $\{w(1)+$ $w(1)-m, \ldots, w(m-1)+w(m-1)-m\} \subseteq S$. Therefore, by applying Proposition 2.1, we have that $S \in \mathscr{C}(m,\{(1,1),(2,2) \ldots,(m-1, m-1)\})$.

To see the other inclusion, let $S \in \mathscr{C}(m,\{(1,1),(2,2) \ldots,(m-1, m-1)\})$ and $x \in S \backslash\{0\}$. On the one hand, if $x \equiv 0(\bmod m)$, then it is clear that $2 x-m \in S$. On the other hand, if $x \not \equiv 0(\bmod m)$, then there exist $i \in\{1, \ldots, m-1\}$ and $t \in \mathbb{N}$ such that $x=w(i)+t m$. Therefore, $2 x-m=(w(i)+w(i)-m)+2 t m \in S$ and in consequence $S \in \mathscr{T}_{m}$.

From Propositions 2.6 and 4.3, we get the following result.

Corollary 4.4 Let $m \in \mathbb{N} \backslash\{0,1\}$. Then $\mathscr{T}_{m}$ is a modular pseudo-Frobenius variety and, in addition, $\Delta(m)$ is the maximum of $S \in \mathscr{T}_{m}$.

In order to build the tree associated with the pseudo-variety $S \in \mathscr{T}_{m}$, we are going to characterise the possible children of each $S \in \mathscr{T}_{m}$.

Proposition 4.5 Let $m \in \mathbb{N} \backslash\{0,1\}, S \in \mathscr{T}_{m}$, and $x \in \operatorname{msg}(S) \backslash\{m\}$. Then $S \backslash\{x\} \in \mathscr{T}_{m}$ if and only if $\frac{x+m}{2} \notin S$.

Proof (Necessity.) If $\frac{x+m}{2} \in S$, then $\frac{x+m}{2} \in S \backslash\{0, x\}$ and $2 \frac{x+m}{2}-m=x \notin S \backslash\{x\}$. Therefore, $S \backslash\{x\} \notin \mathscr{T}_{m}$.

(Sufficiency.) If $a \in S \backslash\{0, x\}$, then $2 a-m \in S$ because $S \in \mathscr{T}_{m}$. Now, if $2 a-m=x$, then $\frac{x+m}{2}=a \in S$. Therefore, $2 a-m \neq x$ and, consequently, $2 a-m \in S \backslash\{x\}$. Thereby, $S \backslash\{x\} \in \mathscr{T}_{m}$.

By applying Theorem 2.7, Propositions 4.3 and 4.5, and Lemma 2.10, we can build the tree $\mathrm{G}\left(\mathscr{T}_{m}\right)$. Let us see an example.

Example 4.6 In the next figure we have the first four levels of $\mathrm{G}\left(\mathscr{T}_{4}\right)$. 


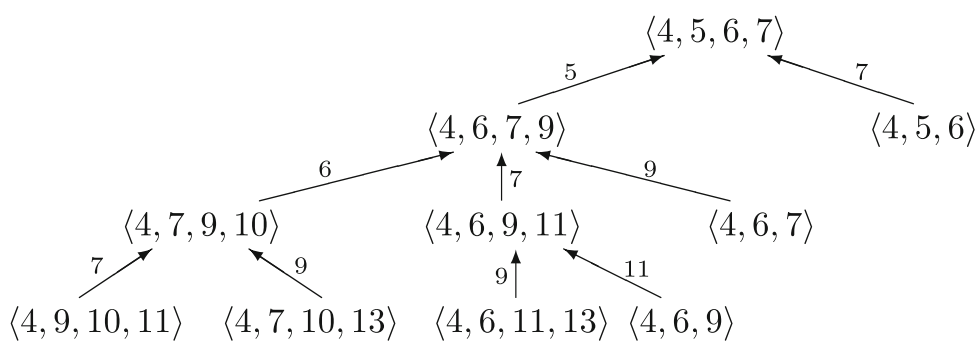

We finish this section describing the Apéry set for $S \in \mathscr{T}_{m}$.

Proposition 4.7 Let $m \in \mathbb{N} \backslash\{0\}, S \in \mathscr{T}_{m}, \operatorname{msg}(S)=\left\{m=n_{1}, n_{2}, \ldots, n_{e}\right\}$. Then $\operatorname{Ap}(S, m)=\{w(0), w(1), \ldots, w(m-1)\}$, where $w(i)$ is the least element of the set $\left\{a_{2} n_{2}+\ldots+a_{e} n_{e} \mid\left(a_{2}, \ldots, a_{e}\right) \in\{0,1\}^{e-1}\right\}$ that is congruent to $i$ modulo $m$.

Corollary 4.8 Let $m \in \mathbb{N} \backslash\{0\}$ and $S \in \mathscr{T}_{m}$. Then $m=\mathrm{m}(S) \leq 2^{\mathrm{e}(S)-1}$.

Remark 4.9 Let us observe that we can generalise the concept of thin numerical semigroups in the following way: setting $n \in \mathbb{N} \backslash\{0,1\}$, we say that a numerical semigroup $S$ is $n$-thin if $n x-\mathrm{m}(S) \in S$ for all $x \in S \backslash\{0\}$, and denote by $\mathscr{T}_{n, m}$ the set of all $k$-thin numerical semigroups with multiplicity equal to $m$. It is clear that $\mathscr{T}_{m}=\mathscr{T}_{2, m}$.

Again, $\mathscr{T}_{n, m}$ is a modular pseudo-variety, Proposition 4.5 is valid for the condition $\frac{x+m}{n} \notin$ $S$, and we can build the chain

$$
\mathscr{T}_{2, m} \subsetneq \mathscr{T}_{3, m} \subsetneq \cdots \subsetneq \mathscr{T}_{m, m}=\mathscr{S}_{m} .
$$

Note that Example 3.14 also gives us the construction that ensures the strict inclusions in this case.

\section{Strong numerical semigroups}

We say that a numerical semigroup $S$ is strong if $x+y-\mathrm{m}(S) \in S$ for all $(x, y) \in(S \backslash\{0\})^{2}$ such that $x \not \equiv y(\bmod \mathrm{m}(S))$. We denote by $\mathscr{R}_{m}$ the set of all the strong numerical semigroups with multiplicity equal to $m$.

Proposition 5.1 Let $S$ be a numerical semigroup with minimal system of generators given by $\left\{m=n_{1}<n_{2}<\cdots<n_{e}\right\}$. Then the following two conditions are equivalents.

1. $S \in \mathscr{R}_{m}$.

2. $\left\{n_{i}+n_{j}-m, 3 n_{i}-m\right\} \subseteq S$ for all $i \in\{2, \ldots, e\}$ and for all $(i, j) \in\{2, \ldots, e\}^{2}$ such that $i \neq j$.

Proof $\left(1 . \Rightarrow 2\right.$.) It is enough to observe that $n_{i} \not \equiv n_{j}(\bmod m)$ and that $2 n_{i} \not \equiv n_{i}(\bmod m)$.

(2. $\Rightarrow$ 1.) Let $x, y \in S \backslash\{0\}$ such that $x \not \equiv y(\bmod m)$. If $x \equiv 0(\bmod m)$ or $y \equiv 0$ $(\bmod m)$, then it is clear that $x+y-m \in S$. Now, if $x \neq \equiv 0(\bmod m)$ and $y \not \equiv 0(\bmod m)$, then there exists $(i, j) \in\{1, \ldots, m-1\}^{2}$, with $i \neq j$, and there exists $(p, q) \in \mathbb{N}^{2}$ such that $x=w(i)+p m$ and $y=w(j)+q m$. Moreover, if there exists $(a, b) \in\{2, \ldots, e\}^{2}$ such that $a \neq b$ and $w(i)-n_{a}, w(j)-n_{b} \in S$, then it is easy to see that $x+y-m \in S$. In other case, there exists $(r, t) \in(\mathbb{N} \backslash\{0\})^{2}$ such that $w(i)=r \cdot n_{a}$ and $w(j)=t \cdot n_{a}$ for some $a \in\{2, \ldots, e\}$. Then, since $i \neq j$, we deduce that $r+t \geq 3$ and, consequently, $x+y-m \in S$. In conclusion, $S \in \mathscr{R}_{m}$. 
The above proposition allows us to easily decide whether a numerical semigroup is strong or not.

Example 5.2 If $S=\langle 4,5,7\rangle$, then $\{5+7-4,3 \cdot 5-4,3 \cdot 7-4\}=\{8,11,17\} \subseteq S$. Therefore, by Proposition 5.1, we have that $S \in \mathscr{R}_{m}$.

Now we want to show that $\mathscr{R}_{m}$ is a modular Frobenius pseudo-variety. Let us denote by $A=\{1, \ldots, m-1\}^{2} \backslash\{(1,1),(2,2), \ldots,(m-1, m-1)\}$.

Proposition 5.3 $\mathscr{R}_{m}=\mathscr{C}(m, A)$ for all $m \in \mathbb{N} \backslash\{0,1\}$.

Proof Let $S \in \mathscr{R}_{m}, \operatorname{Ap}(S, m)=\{w(0), w(1), \ldots, w(m-1)\}$, and $(i, j) \in A$. Then $\left\{(w(i), w(j)\} \in(S \backslash\{0\})^{2}\right.$ and $w(i) \not \equiv w(j)(\bmod m)$. Therefore, $w(i)-w(j)-m \in S$ and, by applying Proposition 2.1, we have that $S \in \mathscr{C}(m, A)$.

To see the other inclusion, let $S \in \mathscr{C}(m, A)$ and $(x, y) \in(S \backslash\{0\})^{2}$. On the one hand, if $0 \in\{x \bmod m, y \bmod m\}$, then it is clear that $x+y-m \in S$. On the other hand, if $0 \notin$ $\{x \bmod m, y \bmod m\}$, then there exist $(i, j) \in A$ and $(p, q) \in \mathbb{N}^{2}$ such that $x=w(i)+p m$ and $y=w(j)+q m$. Therefore, $x+y-m=(w(i)+w(i)-m)+(p+q) m \in S$ and, consequently, $S \in \mathscr{R}_{m}$.

From Propositions 2.6 and 5.3, we get the following result.

Corollary 5.4 Let $m \in \mathbb{N} \backslash\{0,1\}$. Then $\mathscr{R}_{m}$ is a modular pseudo-Frobenius variety and, in addition, $\Delta(m)$ is the maximum of $S \in \mathscr{R}_{m}$.

We are now interested in the description of the tree associated with the pseudo-variety $S \in \mathscr{R}_{m}$. In order to do that, we are going to characterise the children of an arbitrary $S \in \mathscr{R}_{m}$.

Proposition 5.5 Let $m \in \mathbb{N} \backslash\{0,1\}, S \in \mathscr{R}_{m}$, and $x \in \operatorname{msg}(S) \backslash\{m\}$. Then $S \backslash\{x\} \in \mathscr{R}_{m}$ if and only if $x+m \notin\{a+b \mid a, b \in \operatorname{msg}(S) \backslash\{m, x\}, a \neq b\} \cup\{3 a \mid a \in \operatorname{msg}(S) \backslash\{m, x\}\}$.

Proof (Necessity.) If $a, b \in \operatorname{msg}(S) \backslash\{m, x\}$ and $a \neq b$, then $(a, b) \in(S \backslash\{0, x\})^{2}$ and $a \not \equiv b(\bmod m)$. Since $S \backslash\{x\} \in \mathscr{R}_{m}$, we have that $a+b-m \in S \backslash\{x\}$ and, therefore, $a+b-x \neq x$. Thus, $x+m \notin\{a+b \mid a, b \in \operatorname{msg}(S) \backslash\{m, x\}, a \neq b\}$.

On the other hand, if $a \in \operatorname{msg}(S) \backslash\{m, x\}$, then $(a, 2 a) \in(S \backslash\{0, x\})^{2}$ and $a \not \equiv 2 a$ $(\bmod m)$. Once again, since $S \backslash\{x\} \in \mathscr{R}_{m}$, we have that $3 a-m \in S \backslash\{x\}$ and, therefore, $a+b-x \neq x$. Thus, $x+m \notin\{3 a \mid a \in \operatorname{msg}(S) \backslash\{m, x\}\}$.

(Sufficiency.) Let $a, b \in S \backslash\{0, x\}$ such that $a \neq b$. Since $S \in \mathscr{R}_{m}$, we have that $a+b-m \in$ $S$ and $3 a-m \in S$. Now, by Lemma 2.10, we know that $\operatorname{msg}(S) \backslash\{x\} \subseteq \operatorname{msg}(S \backslash\{x\}) \subseteq$ $(\operatorname{msg}(S) \backslash\{x\}) \cup\{x+m\}$. Thus, from this fact and the hypothesis, it easily follows that $a+b-m \neq x$ and $3 a-m \neq x$, that is, $a+b-m, 3 a-m \in S \backslash\{x\}$. By applying Proposition 5.1, we conclude that $S \backslash\{x\} \in \mathscr{R}_{m}$.

From Theorem 2.7, Propositions 5.3 and 5.5, and Lemma 2.10, we can build the tree $\mathrm{G}\left(\mathscr{R}_{m}\right)$. Let us see an example.

Example 5.6 In the next figure we have the first four levels of $\mathrm{G}\left(\mathscr{R}_{4}\right)$.

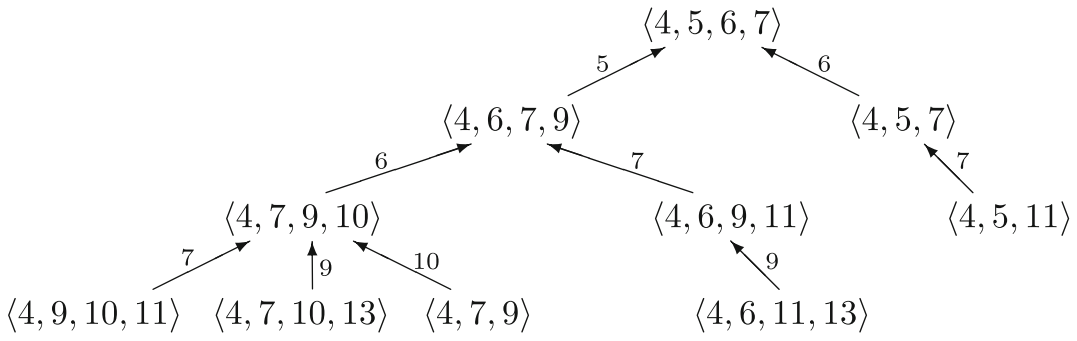


We finish this section describing the Apéry set for $S \in \mathscr{R}_{m}$.

Proposition 5.7 Let $m \in \mathbb{N} \backslash\{0,1\}, S \in \mathscr{R}_{m}, \operatorname{msg}(S)=\left\{m=n_{1}, n_{2}, \ldots, n_{e}\right\}$. Then $\operatorname{Ap}(S)=\{w(0), w(1), \ldots, w(n)\}$, where $w(i)$ is the least element of the set $\left\{0, n_{2}, \ldots, n_{e}\right\} \cup$ $\left\{2 n_{2}, \ldots, 2 n_{e}\right\}$ that is congruent to $i$ modulo $m$.

Corollary 5.8 Let $m \in \mathbb{N} \backslash\{0,1\}$ and $S \in \mathscr{R}_{m}$. Then $m=\mathrm{m}(S) \leq 2 \mathrm{e}(S)-1$.

It is interesting to observe that, under the hypotheses of Proposition 5.7, $n_{i} \in$ Maximals $_{\leq S}(\operatorname{Ap}(S, m))$ if and only if $2 n_{i} \notin \operatorname{Ap}(S, m)$. In addition, $2 n_{i} \in \operatorname{Ap}(S, m)$ if and only if $2 n_{i} \in$ Maximals $_{\leq S}(\operatorname{Ap}(S, m))$. Therefore, from Propositions 3.11 and 5.7, we get the next result.

Corollary 5.9 Let $m \in \mathbb{N} \backslash\{0,1\}$ and $S \in \mathscr{R}_{m}$. Then $\mathrm{t}(S)=\mathrm{e}(S)-1$.

It is well known that, if $S$ is a numerical semigroup, then $(S) \leq \mathrm{m}(S)$ and $\mathrm{t}(S) \leq \mathrm{m}(S)-1$ (see Proposition 2.10 and Corollary 2.23 in [11]). Combining these facts with Corollaries 5.8 and 5.9, we have the next result.

Corollary 5.10 Let $m \in \mathbb{N} \backslash\{0,1\}$ and $S \in \mathscr{R}_{m}$. Then $\frac{\mathrm{m}(S)-1}{2} \leq \mathrm{t}(S) \leq \mathrm{m}(S)-1$ (or, equivalently, $\left.\frac{\mathrm{m}(S)+1}{2} \leq \mathrm{e}(S) \leq \mathrm{m}(S)\right)$.

Remark 5.11 Contrary to what happens in Sects. 3 and 4, the concept of strong numerical semigroup does not have a natural generalisation. In fact, we have, at least, two possibilities.

1. $x_{1}+\cdots x_{n}-\mathrm{m}(S) \in S$ for all $\left(x_{1}, \ldots, x_{n}\right) \in(S \backslash\{0\})^{n}$ such that $x_{i} \not \equiv x_{j}(\bmod \mathrm{m}(S))$, for all $i \neq j$. In this case, $\left(x_{1}, \ldots, x_{n}\right) \in A_{n}$, where

$$
A_{n}=\left\{\left(\alpha_{1}, \ldots, \alpha_{n}\right) \in\{1, \ldots, m-1\}^{n} \mid \alpha_{i} \neq \alpha_{j} \text { for all } i \neq j\right\} .
$$

2. $x_{1}+\cdots x_{n}-\mathrm{m}(S) \in S$ for all $\left(x_{1}, \ldots, x_{n}\right) \in B_{n}$, where

$$
B_{n}=\{1, \ldots, m-1\}^{n} \backslash\{(1, \ldots, 1),(2, \ldots, 2), \ldots,(m-1, \ldots, m-1)\} .
$$

It is clear that, if $n \geq 3$, then $A_{n} \subsetneq B_{n}$ and, in consequence, $\mathscr{C}\left(m, B_{n}\right) \subseteq \mathscr{C}\left(m, A_{n}\right)$. In addition, it is guessed that, if $n<m$, then the second inclusion will be strict.

Acknowledgements The authors thank the referee for the helpful comments and suggestions which have improved this paper.

Funding Funding for open access charge: Universidad de Granada / CBUA

Open Access This article is licensed under a Creative Commons Attribution 4.0 International License, which permits use, sharing, adaptation, distribution and reproduction in any medium or format, as long as you give appropriate credit to the original author(s) and the source, provide a link to the Creative Commons licence, and indicate if changes were made. The images or other third party material in this article are included in the article's Creative Commons licence, unless indicated otherwise in a credit line to the material. If material is not included in the article's Creative Commons licence and your intended use is not permitted by statutory regulation or exceeds the permitted use, you will need to obtain permission directly from the copyright holder. To view a copy of this licence, visit http://creativecommons.org/licenses/by/4.0/.

\section{References}

1. Apéry, R.: Sur les branches superlinéaires des courbes algébriques. C. R. Acad. Sci. Paris 222, 1198-1200 (1946) 
2. Barucci, V., Dobbs, D.E., Fontana, M.: Maximality Properties in Numerical Semigroups and Applications to One-Dimensional Analytically Irreducible Local Domains, Mem. Amer. Math. Soc. 598 (1997)

3. Bras-Amorós, M., García-Sánchez, P.A.: Patterns on numerical semigroups. Linear Algebra Appl. 414, 652-669 (2006)

4. Bras-Amorós, M., García-Sánchez, P.A., Vico-Oton, A.: Nonhomogeneous patterns on numerical semigroups. Int. J. Algebra Comput. 23, 1469-1483 (2013)

5. Campillo, A., Farrán, J.I., Munuera, C.: On the parameters of algebraic-geometry codes related to Arf semigroups. IEEE Trans. Inform. Theory 46(7), 2634-2638 (2000)

6. Delgado, M., García-Sánchez, P.A., Morais, J.: NumericalSgps, a GAP package for numerical semigroups, version 1.2.2 (03/03/2020). https://gap-packages.github.io/numericalsgps/

7. Ramírez Alfonsín, J.L.: The Diophantine Frobenius problem. In: Oxford Lectures Series in Mathematics and its Applications, vol. 30. Oxford Univ. Press (2005)

8. Robles-Pérez, A.M., Rosales, J.C.: The numerical semigroup of phrases’ lengths in a simple alphabet, The Scientific World Journal 2013 (2013), Article ID 459024, 9 pages

9. Robles-Pérez, A.M., Rosales, J.C.: Frobenius pseudo-varieties in numerical semigroups. Ann. Mat. Pura Appl. 194, 275-287 (2015)

10. Rosales, J.C., Branco, M.B.: Numerical semigroups that can be expressed as an intersection of symmetric numerical semigroups. J. Pure Appl. Algebra 171, 303-314 (2002)

11. Rosales, J.C., García-Sánchez, P.A.: Numerical semigroups, Developments in Mathematics, vol. 20. Springer, New York (2009)

12. Rosen, K.H.: Handbook of Discrete and Combinatorial Mathematics. CRC Press, Boca Raton (2000)

13. Selmer, E.S.: On the linear diophantine problem of Frobenius. J. Reine Angew. Math. 293(294), 1-17 (1977)

14. Stokes, K.: Patterns of ideals numerical semigroups. Semigroup Forum 93, 180-200 (2016)

15. Sylvester, J.J.: Problem 7382, The Educational Times, and Journal of the College of Preceptors, New Ser., 36(266) (1883), 177. Solution by W. J. Curran Sharp, ibid., 36(271) (1883), 315

Publisher's Note Springer Nature remains neutral with regard to jurisdictional claims in published maps and institutional affiliations. 